Pacific Journal of Mathematics

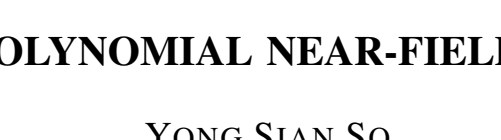




\section{POLYNOMIAL NEAR-FIELDS?}

\section{YONG-SIAN So}

It is well known that all finite fields can be obtained as homomorphic images of polynomial rings. Hence it is natural to raise the question, which near-fields arise as homomorphic images of polynomial near-rings.

It is the purpose of this paper to give the surprising answer: one gets no proper near-fields at all-in dramatic contrast to ring and field theory. Another surprising result is the fact that all near-fields contained in the near-rings of polynomials are actually fields.

Homomorphic images are essentially factor structures. So we take a commutative ring $R$ with identity, from the near-ring $R[x]$ of all polynomials over $R$ (or the near-ring $R_{0}[x]$ of all polynomials without constant term over $R$ ) and look for ideals $I$ such that $R[x] / I$ becomes a near field. With this notation (and containing the one of [1] and [2]) we get our main result:

THEOREM 1. If $R[x] / I$ (or $R_{0}[x] / I$ ) is a near-field then it is isomorphic to $R / M$ (where $M$ is a maximal ideal of $R$ ) and hence a field.

The proof requires a series of lemmas as well as a number of results on near-fields.

Our first reduction is the one of $R[x]$ to $R_{0}[x]$.

LEMMA 1. If $I$ is an ideal of (the near-ring) $R[x]$ such that $R[x] / I$ is a near-field, then there exists an ideal $J$ of $R_{0}[x]$ with $R[x] / I \cong R_{0}[x] / J$.

Proof. $R_{0}[x] \leqq I$ implies $x \in I$, hence $R[x] \leqq I$, a contradiction. So we have $R_{0}[x] \nsubseteq I$ and-since $I$ must be maximal in order to get a near-field- $R_{0}[x]+I=R[x]$. By a version of the isomorphic theorem (which is valid in our case) we get

$$
R[x] / I=\left(R_{0}[x]+I\right) / I \cong R_{0}[x] /\left(I \cap R_{0}[x]\right)
$$

and $J:=R_{0}[x] \cap I$ will do the job.

REMARK 1. The converse of Lemma 1 does not hold: Take $J:=\left\{a_{2} x^{2}+a_{3} x^{3}+\cdots+a_{n} x^{n} / n \in N, n \geqq 2, a_{i} \in \boldsymbol{R}\right\}$. Then $\boldsymbol{R}_{0}[x] / J \cong \boldsymbol{R}$ is a (near) field, but the near-ring $R[x]$ is simple ([2] or [3], 7.89), so there is no $I \leqq \boldsymbol{R}[x]$ with $\boldsymbol{R}[x] / I \cong \boldsymbol{R}$. 
We can therefore reduce our search to get suitable ideals of $R_{0}[x]$ which yield near-field factors.

LeMmA 2. Let $I \leqq R_{0}[x]=: N$. Then $R_{0}[x] / I$ is a near-field iff $I$ is a maximal $N$-subgroup of $N$.

Proof. $\Rightarrow$ : Suppose that $N / I$ is a near-field. Then $N / I$ is $N / I$ simple by ([3], 8.3). Consider the canonical epimorphism $h: N \rightarrow N / I$ with kernel $I$. If $M$ is some $N$-subgroup strictly between $I$ and $N$ then $h(M)$ turns out to be a proper $N / I$-subgroup of $N / I$, which is a contradiction. Hence $I$ is a maximal $N$-subgroup of $N$.

$\Longleftarrow$ : Let $I$ be a maximal $N$-subgroup of $N$ and take $h$ as above. If $M$ is a proper $N / I$-subgroup of $N / I$ then $h^{-1}(M)$ is an $N$-subgroup of $N$ strictly between $I$ and $N$, which cannot happen. Hence $N / I$ is $N / I$-simple and again by ([3], 8.3) a near-field.

Due to the works of Clay-Doi [2], Brenner [1] and Straus [5] we know quite a bit about maximal ideals of $R[x]$. These informations can be used to find all ideals $I$ of $R_{0}[x]$ which are maximal $R_{0}[x]$ subgroups of $R_{0}[x]$ and which we call "strictly maximal" ones (from now on).

First we need some

Notations.

(i ) $\left(\left(x^{2}\right)\right):=\left\{a_{2} x^{2}+\cdots+a_{n} x^{n} / n \in N, n \geqq 2, a_{i} \in R\right\}$.

(ii) If $I \leqq R_{0}[x]$ then $I_{1}:=\left\{a \in R /\right.$ some $\left.a x+a_{2} x^{2}+\cdots+a_{n} x^{n} \in I\right\}$ $I^{\prime}:=\{a \in R / a x \in I\}$.

(iii) If $M \triangleleft R$ then $M x:=\{m x / m \in M\}$.

LEMMA 3. ( i ) $\left(\left(x^{2}\right)\right)$ is an ideal of $R_{0}[x]$ with $R_{0}[x] /\left(\left(x^{2}\right)\right) \cong R$.

(ii) $I_{1}$ and $I^{\prime}$ are ideals of $R$ with $I^{\prime} \subseteq I_{1}$.

Proof. Straightforward.

Lemma 4. Let I be a strictly maximal ideal of $R_{0}[x]$ and $h: R \rightarrow$ $R / I^{\prime}$ the canonical epimorphism. We define $h^{\prime}$ as follows: $h^{\prime}: R_{0}[x] \rightarrow$ $\left(R / I^{\prime}\right)_{0}[x]$

$$
a_{n} x^{n}+\cdots+a_{1} x \longmapsto h\left(a_{n}\right) x^{n}+\cdots+h\left(a_{1}\right) x .
$$

Then $J:=h^{\prime}(I)$ is a strictly maximal ideal in $\left(R / I^{\prime}\right)_{0}[x]=h^{\prime}\left(R_{0}[x]\right)$ and $J^{\prime}$ is the zero ideal in $R / I^{\prime}$.

Proof. By ([4], 4.6), $h^{\prime}$ is a near-ring epimorphism and we get 
$R_{0}[x] / I \cong h^{\prime}\left(R_{0}[x]\right) / h^{\prime}(I)=\left(R / I^{\prime}\right)_{0}[x] / J$. So $J$ must be strictly maximal in $\left(R / I^{\prime}\right)_{0}[x]$, by arguments as in Lemma 2. Observe that $\left(I^{\prime}\right)_{0}[x] \subseteq I$.

Now suppose that $r^{\prime} \in R / I^{\prime}$ is in $J^{\prime}$. Then $r^{\prime} x \in J=h^{\prime}(I)$ and there is some $i \in I$ with $h^{\prime}(i)=r^{\prime} x$. Let $i=a_{1} x+\cdots+a_{n} x^{n}$. Then $h^{\prime}(i)=$ $h\left(a_{1}\right) x+\cdots+h\left(a_{n}\right) x^{n}=r^{\prime} x$, whence $-r x+a_{1} x+\cdots+a_{n} x^{n} \in \operatorname{Ker} h^{\prime}=$ $\left(I^{\prime}\right)_{0}[x] \leqq I$ for some $r \in R$ with $h(r)=r^{\prime}$. Hence $r x$ must be in $I$, so $r \in I^{\prime}$ and consequently $r^{\prime}$ is the zero element of $R / I^{\prime}$. This shows that $J^{\prime}$ is the zero ideal of $R / I^{\prime}$.

By using the second isomorphism theorem, we therefore can confine our attention to strictly maximal ideals $I$ with $I^{\prime}=\{0\}$. But then the worst cases are behind of us:

Lemma 5. Let $I$ be a strictly maximal ideal in $R_{0}[x]$ with $I^{\prime}=$ $\{0\}$. Then $R$ is an integral domain.

Proof. Let $a, b \in R, a \neq 0, b \neq 0$ and $a b=0$. Then $a x \circ b x=$ $a b x=0 \in I$. If both $a x \notin I, b x \notin I$ then $(a x+I) \circ(b x+I)=a b x+I=I$; a contradiction to the fact that a near-field has no divisors of zero. So we get $a x \in I$ or $b x \in I$, whence $a \in I^{\prime}$ or $b \in I^{\prime}$, a contradiction. $R$ is therefore an integral domain.

By ([3], 8.9), the characteristic of a near-field is either 0 , a prime $\neq 2$ or $=2$. We treat these 3 cases separately, and start with:

LEMMA 6. Let $I$ be a strictly maximal ideal of $R_{0}[x]$ with $I^{\prime}=$ $\{0\}$ and Char $R_{0}[x] / I=0$. Then there exists a maximal ideal $M$ of $R$ with $R_{0}[x] / I=R / M$.

Proof. By Lemma 5, $R$ is an integral domain. It is easy to see that in our case Char $R=$ Char $R_{0}[x]=$ Char $R_{0}[x] / I=0$, hence $R$ is infinite.

Case 1. $\left(\left(x^{2}\right)\right) \subseteq I$. Since $I_{1}$ cannot be $=R$ (otherwise $\left.I=R_{0}[x]\right)$, $I_{1}$ is contained in a maximal ideal $M$ of $R$. $I=\left(\left(x^{2}\right)\right)+I_{1} x \leqq\left(\left(x^{2}\right)\right)+$ $M x$ which is a proper ideal of $R_{0}[x]$. But $I$ is a strictly maximal ideal, hence $I=\left(\left(x^{2}\right)\right)+M x$ and $R_{0}[x] / I \cong(\{a x / a \in R / M\},+, 0) \cong(R / M,+, \cdot)$.

Case 2. $\left(\left(x^{2}\right)\right) \nsubseteq I$. Since $I$ is a strictly maximal ideal we get $I+\left(\left(x^{2}\right)\right)=R_{0}[x]$. Then $I_{1}=R$ and we can select a polynomial $i=$ $b_{n} x^{n}+\cdots+b_{1} x \in I$ with $b_{1} \neq 0$ and $n$ minimal for being a polynomial in $I$ with nonzero coefficient of $x$. If $r \in R$ then $i \circ(r x)-r x \circ i \in I-$ $I=I$. But $i \circ(r x)-r x \circ i=b_{n-1}\left(r^{n}-r^{n-1}\right) x^{n-1}+\cdots+b_{2}\left(r^{n}-r^{2}\right) x^{2}+$ 
$b_{1}\left(r^{n}-r\right) x$. Since $R$ is an integral domain, hence embeddable into a field, the set of all $s \in R$ with $s^{n}=s$ has cardinality $\leqq n$. Since $R$ is infinite, we can take $r \in R$ so that $r^{n} \neq r$. Then $i \circ(r x)-r x \circ i$ is a polynomial in $I$ with nonzero coefficient of $x$ and a degree $\leqq n-1$ which is a contradiction. So Case 2 cannot occur.

Hence we have proved our Theorem 1 in the case when Char $R_{0}[x] / I=0$. Now we consider the case of characteristic $p \neq 2$.

Lemma 7. Let $I$ be a strictly maximal ideal of $R_{0}[x]$ with Char $R_{0}[x] / I \neq 2$. Then there exists a maximal ideal $M$ of $R$ with $I=M x+\left(\left(x^{2}\right)\right)$, hence $R_{0}[x] / I \cong R / M$.

Proof. First we show: $x^{2} \in I$. Since $x \notin I,-x \notin I$. If $x^{2} \notin I$ we have: $\left(x^{2}+I\right) \circ(-x+I)=-\left(\left(x^{2}+I\right) \circ(x+I)\right)=-\left(x^{2}+I\right)=-x^{2}+I$ by $([3], 8.10(\mathrm{~b}))$. But $\left(x^{2}+I\right) \circ(-x+I)=x^{2} \circ(-x)+I=x^{2}+I$. So we have $2 x^{2} \in I$. Since $(p, 2)=1$ there are $a, b \in Z$ with $1=a \cdot p+$ $b \cdot 2$. $x^{2}=(a \cdot p+b \cdot 2) x^{2}=a p x^{2}+2 b x^{2} \in I$ because $p x^{2} \in I$ as a result of Char $R_{0}[x] / I=p$. This is contradiction, hence $x^{2} \in I$. Then we have $x^{2 n}=x^{2} \circ x^{n} \in I$ for all $n \in N$.

Now we show: $x^{n} \in I$ for all $n \in N$ and $n \geqq 2$. Let $n \geqq 2$. Then $x^{2} \circ\left(x^{n}+x^{n-1}\right)=x^{2 n}+2 x^{2 n-1}+x^{2 n-2} \in I$, and we get $2 x^{2 n-1} \in I$ because $x^{2 n} \in I$ for $n \geqq 1$. As above, we have $x^{2 n-1} \in I$. Hence we have: $x^{n} \in I$ for $n \geqq 2$. And as a result of this we have $\left(\left(x^{2}\right)\right) \subseteq I$ and, similarly to the proof of Lemma 6 , we have $I=M x+\left(\left(x^{2}\right)\right)$ where $M$ is a maximal ideal of $R$. Therefore $R_{0}[x] / I \cong R / M$.

So it remains the case that $\operatorname{Char} R_{0}[x] / I=2$, which-as usualcauses the most trouble.

LEMMA 8. Let $I$ be a strictly maximal ideal in $R_{0}[x]$ with Char $R_{0}[x] / I=2$. Then $(2 R)_{0}[x] \subseteq I$.

Proof. Since $x+I \in R_{0}[x] / I$ we have $2 x+I=I$. Hence $2 x \in I$. But for all $f \in R_{0}[x] 2 x \circ f=2 f \in I$, hence $(2 R)_{0}[x] \subseteq I$.

LeMma 9. Let $I$ be a strictly maximal ideal in $R_{0}[x]$ with Char $R_{0}[x] / I=2$. Also, let $h: R \rightarrow R / 2 R$ be the canonical epimorphism and $h^{\prime}: R_{0}[x] \rightarrow(R / 2 R)_{0}[x]: a_{n} x^{n}+\cdots+a_{1} x \rightarrow h\left(a_{n}\right) x^{n}+\cdots+h\left(a_{1}\right) x$. Then $R_{0}[x] / I \cong(R / 2 R)_{0}[x] / h^{\prime}(I)$.

The proof is similar to the one of Lemma 4 and therefore omitted. In view of this result, we only have to look at the case: Char $R=$ Char $R_{0}[x] / I=2, R$ an integral domain and $I^{\prime}=\{0\}$. 
We now treat the infinite case:

Lemma 10. Let $I$ be a strictly maximal ideal in $R_{0}[x]$ with Char $R=$ Char $R_{0}[x] / I=2, R$ an infinite integral domain and $I^{\prime}=\{0\}$. Then there exists a maximal ideal $M$ of $R$ with $I=\left(\left(x^{2}\right)\right)+M x$, hence $R_{0}[x] / I=R / M$.

Proof. Suppose there is no maximal ideal $M$ of $R$ with $I=$ $\left(\left(x^{2}\right)\right)+M x$. Then we get $I_{1}=R$, otherwise $I_{1}$ would be in a maximal ideal $M_{1}$ of $R$ and $I \subseteq\left(\left(x^{2}\right)\right)+M_{1} x$.

Let $U:=\left\{a_{n} x^{n}+\cdots+a_{1} x \in I / n \in N, a_{1} \neq 0\right\}$. Clearly $U \neq\{0\}$, since $I_{1}=R$. Let $m$ be the minimum of the degrees of nonzero polynomials in $U$. Since $I^{\prime}=\{0\}, m$ is $\geqq 2$. Let $e \in R \backslash\{0,1\} \neq \varnothing$. Let $b_{m} x^{m}+\cdots+b_{1} x \in U \subseteq I . \quad\left(b_{m} x^{m}+\cdots+b_{1} x\right) \circ(e x)+e^{m} x \circ\left(b_{m} x^{m}+\cdots\right.$ $\left.+b_{1} x\right)=b_{m-1}\left(e^{m}+e^{m-1}\right) x^{m-1}+\cdots+b_{1}\left(e^{m}+e\right) x \in I$. Since $m$ is minimal, $b_{1}\left(e^{m}+e\right)=0$. We get $e^{m}+e=0, e^{m-1}+1=0$, because $R$ is an integral domain. But $1^{m-1}+1=0$, so we get for all $e \in R \backslash\{0\}$ $e^{m-1}+1=0$.

So $m-2 \geqq 1$; consequently $e^{m-1}=e \cdot e^{m-2}=1$ and hence $e^{m-2}$ is the inverse of $e$ in $R$. $R$ is then a field with $e^{m-1}=1$ for all $e \in R \backslash\{0\}$, hence with infinitely many roots of unity, a contradiction.

So there is a maximal ideal $M$ of $R$ with $I=\left(\left(x^{2}\right)\right)+M x$.

In particular, if $R$ is a field, we get $I=\left(\left(x^{2}\right)\right)$.

We still have to look at the case: Char $R=2, R$ a finite integral domain, $I^{\prime}=\{0\}$. But a finite integral domain is a field. So for our $R$ we have either $R=Z_{2}$ or $R=G F\left(2^{n}\right)$ with $n \geqq 2$.

First some preparations:

Lemma 11. Let $F$ be a field with Char $F=2,|F|>2$. Let $I$ be a strictly maximal ideal in $F_{0}[x]$. If $x^{m} \in I$ then $x^{m+i} \in I$ for $m+i \geqq 4$ where $i \in N$.

Proof. $x^{2 m+1}+x^{m+2}=\left(x^{m}+x\right)^{3}+x^{3}+x^{3 m} \in I$. Since $|F|>2$, it is possible to choose $a$ with $a \neq 0, a \neq 1$. From $\left(x^{m}+a x\right)^{3}+(a x)^{3} \in I$ we get $a x^{2 m+1}+a^{2} x^{m+2} \in I$. But $a x \circ\left(x^{2 m+1}+x^{m+2}\right)=a x^{2 m+1}+a x^{m+2} \in I$. By adding of these two polynomials we get $\left(a^{2}+a\right) x^{m+2} \in I$. Since $a^{2}+a \neq 0$, we have $x^{m+2} \in I$. So we have: $x^{m}, x^{m+2}, x^{m+4}, x^{m+6}, \cdots \in I$.

But $x^{2 m}=x^{m} \circ x^{2} \in I$, we also have $x^{2 m+2} \in I . \quad x^{2 m+2}=\left(x^{m+1}\right) \circ x^{2} \in I$, so we have either $x^{2} \in I$ or $x^{m+1} \in I$ since $F_{0}[x] / I$ is a near-field and has no zero-divisor.

If $x^{m+1} \in I$ we get: $x^{m+i} \in I$ for all $i \in N$.

If $x^{2} \in I$ then $x^{4}+x^{5}=\left(x^{2}+x\right)^{3}+x^{3}+x^{6} \in I$. Hence then $x^{5} \in I$. 
So we have: $x^{2}, x^{4}, x^{6}, \cdots \in I, x^{5}, x^{7}, x^{9}, \cdots \in I$.

Hence $x^{m+i} \in I$ for $m+i \geqq 4$, where $i \in N$.

Lemma 12. Let $I \neq F_{0}[x]$ be an ideal of $F_{0}[x]$, when $F$ is a field of characteristic 2 . If there is an $n \geqq 2$, so that $x^{m} \in I$ for all $m \geqq n$, then $I \subseteq\left(\left(x^{2}\right)\right)$.

Proof. Suppose $I \nsubseteq\left(\left(x^{2}\right)\right)$. Then there is some $f \in I \backslash\left(\left(x^{2}\right)\right)$. Without loss of generality, we can assume $f=x+a_{2} x^{2}+\cdots+a_{n-1} x^{n-1}$.

$$
\begin{aligned}
& f \circ x^{n-1}=x^{n-1}+a^{2}\left(x^{n-1}\right)^{2}+\cdots+a_{n-1}\left(x^{n-1}\right)^{n-1} \in I \\
& x^{n-1}=f \circ x^{n-1}+a_{2}\left(x^{n-1}\right)^{2}+\cdots+a_{n-1}\left(x^{n-1}\right)^{n-1} \in I
\end{aligned}
$$

since the degrees of second, third, $\cdots$ terms are $\geqq n$. Therefore we can reduce $n$ and we get: $x^{n-2}, x^{n-3}, \cdots, x^{2} \in I$. But then $x=f+$ $a_{2} x^{2}+\cdots+a_{n-1} x^{n-1} \in I$, a contradiction. Hence $I \subseteq\left(\left(x^{2}\right)\right)$.

LEMma 13. Let $I$ be a maximal ideal in $F_{0}[x]$, when $F$ is a field of characteristic 2 and $|F|>2$. If there is some $n \in N$ with $n \geqq 2$, so that $x^{m} \in I$ for all $m \geqq n$, then $I=\left(\left(x^{2}\right)\right)$.

\section{Proof. Use Lemma 12.}

LEMMA 14. Let $I$ be a strictly maximal ideal in $F_{0}[x]$, when $F$ is a field of characteristic 2 and $|F|>2$. If there is an $n \in N$ with $n \geqq 2, x^{n} \in I$, then $I=\left(\left(x^{2}\right)\right)$.

Proof. According to Lemma 11 we have: $x^{m} \in I$ for all $m \geqq$ $\max (n, 4)$. Lemma 13 will do the rest of the job.

Lemma 15. Let $F$ be a field of characteristic 2 and $I$ a strictly maximal ideal of $F_{0}[x]$. Then there is an odd number $t$ with $x^{t}+\cdots+a_{1} x \in I$.

Proof. Since $I \neq\{0\}$, there is a $k \in N$ with $x^{2 k}+\cdots+b_{1} x \in I$, otherwise our assertion is already proved.

$\left(x^{2 k}+\cdots+b_{1} x+x\right)^{3}+x^{3}=\left(x^{2 k}+\cdots+b_{1} x\right)^{3}+\left(x^{2 k}+\cdots+b_{1} x\right)^{2} x+$ $\left(x^{2 k}+\cdots+b_{1} x\right) x^{2} \in I$. We get $x^{4 k+1}+\cdots+x^{2 k+2}+\cdots \in I$. For $n \geqq 1$, $4 k+1$ is greater than $2 k+2$ and so there is a polynomial of degree $4 k+1$ (an odd number) in $I$.

LEMma 16. Let $F$ be a finite field of characteristic 2 and $I$ a strictly maximal ideal of $F_{0}[x]$. Then the near-field $F_{0}[x] / I$ is finite.

Proof. We know from Lemma 15 that there is an odd number 
$t$ with $x^{t}+\cdots+a_{1} x \in I$.

We show: For all $n \geqq 6 t$ there is some $x^{n}+\cdots+b_{1} x \in I$.

For all $l \geqq 1,\left(x^{t}+\cdots+a_{1} x+x^{t+l}\right)^{3}+\left(x^{t+l}\right)^{3} \in I$. Hence $\left(x^{t+l}\right)^{2}\left(x^{t}+\cdots\right.$ $\left.+a_{1} x\right)+\left(x^{t+l}\right)\left(x^{t}+\cdots+b_{1} x\right)^{2} \in I$, whence $x^{3 t+2 l}+\cdots+x^{3 t+l}+\cdots \in I$. Since $\left(x^{t}+\cdots+a_{1} x\right)^{3}=x^{3 t}+\cdots \in I$, there are polynomials of following degrees in $I: 3 t, 3 t+2,3 t+4, \cdots$. Since $3 t$ is odd, we have: For all odd numbers $k \geqq 3 t$, there is some normed polynomial of degree $k$ in $I$.

$$
\begin{aligned}
& \left(x^{t}+\cdots+a_{1} x\right)^{6}=x^{6 t}+\cdots \in I . \\
& \left(x^{t}+\cdots+a_{1} x\right)^{2}=x^{2 t}+\cdots+e_{1} x \in I . \\
& \left(x^{2 t+l}+x^{2 t}+\cdots+e_{1} x\right)^{3}+\left(x^{2 t+l}\right)^{3} \in I .
\end{aligned}
$$

Hence $\left(x^{2 t+l}\right)^{2}\left(x^{2 t}+\cdots\right)+\left(x^{2 t+l}\right)\left(x^{2 t}+\cdots\right)^{2} \in I$, whence $x^{6 t+2 l}+\cdots+$ $x^{6 t+l}+\cdots \in I$. Therefore there are also polynomials of following degrees in $I: 6 t, 6 t+2,6 t+4, \cdots$.

We get: For all $k \geqq 6 t$ there exists some polynomial $x^{k}+\cdots+$ $b_{1} x \in I$. Hence $\left|F_{0}[x] / I\right| \leqq|F|^{6 t}$, which is finite.

Lemma 17. Let $F$ be $G F\left(2^{n}\right), n \geqq 2$ and $I$ a strictly maximal ideal of $F_{0}[x]$. Then $I=\left(\left(x^{2}\right)\right)$.

Proof. Lemma 16 tells us that $K:=F_{0}[x] / I$ is a finite near-field. By 8.34 of [3], all finite near-fields (except 7 exceptional cases of orders $\left.5^{2}, 11^{2}, 7^{2}, 23^{2}, 11^{2}, 29^{2}, 59^{2}\right)$ are Dikson near-fields. Our $K$ cannot be exceptional, so it is a Dickson near-field. In this case, we know from 3.3 of [6] that the center $C(K):=\{f \in K / f \circ g=g \circ f$ for all $g \in K\}$ is closed with respect to addition.

Since, by the well-known rules how to calculate in $G F\left(2^{n}\right), x+I$ and $x^{2^{n}}+I$ belong to $C(K)$, so does their sum $x+x^{2^{n}}+I$. So we get $\left(x^{2^{n}}+x+I\right) \circ\left(x^{2^{n}-1}+I\right)=\left(x^{2^{n}-1}+I\right) \circ\left(x^{2^{n}}+x+I\right) . \quad\left(x^{2^{n}-1}\right)^{2^{n}}+$ $x^{2^{n}-1}+I=\left(x^{2^{n}}+x\right)^{2^{n}-1}+I=\left(x^{2^{n}}\right)^{2^{n}-1}+\left(x^{2^{n}}\right)^{2^{n}-2}+\cdots+x^{2^{n}} x^{2^{n}-2}+x^{2^{n}-1}+$ $I=x^{\left(2^{n}-1\right) 2^{n}}+\sum_{k=1}^{2^{n}-2} x^{2^{n} k+\left(2^{n}-1-k\right)}+x^{2^{n}-1}+I$. Hence $\sum_{k=1}^{2^{n}-2} x^{\left.2^{2_{k+(2 n}}-1-k\right)} \in I$. But $2^{n} k+\left(2^{n}-1-k\right)=\left(2^{n}-1\right) k+\left(2^{n}-1\right)=\left(2^{n}-1\right)(k+1)$, so $\sum_{k=1}^{2^{n}-2} x^{\left(2^{n}-1\right)(k+1)}=\sum_{k=1}^{2^{n}-2}\left(x^{2^{n}-1}\right)^{k+1}=\left(\sum_{k=1}^{2^{n}-2} x^{k+1}\right) \circ x^{2^{n}-1} \in I$. Since $K$ is a near-field, either $\sum_{k=1}^{2^{n}-2} x^{k+1} \in I$ or $x^{2^{n}-1} \in I$. If $x^{2^{n}-1} \in I$, we are through, for we get $I=\left(\left(x^{2}\right)\right)$ by Lemma 14 . So we may assume that $\sum_{k=1}^{2^{n}-2} x^{k+1}=x^{2^{n}-1}+\cdots+x^{2} \in I$.

The multiplicative group of $G F\left(2^{n}\right)$ is cyclic. Therefore there is some $c \in G F\left(2^{n}\right)$ of order $2^{n}-1$. We know: $c \neq 0, c \neq 1 . \quad c^{2^{n}-1}=1$ and for all $l<2^{n}-1 c^{l} \neq 1$ and for all $l, j \leqq 2^{n}-1, l \neq j: c^{l}+c^{j} \neq 0$. Since $c^{2^{n}-1} x^{2^{n}-1}+\cdots+c x^{2}=\left(x^{2^{n}-1}+\cdots+x^{2}\right) \circ(c x) \in I, c^{2^{n}-1} x^{2^{n}-1}+\cdots$ $+c^{2^{n}-1} x^{2}=c^{2^{n}-1} x \circ\left(x^{2^{n}-1}+\cdots+x^{2}\right) \in I$, we get $\left(c^{2^{n}-1}+c^{2^{n}-2}\right) x^{2^{n}-2}+\cdots$ 
$+\left(c^{2^{n}-1}+c^{2}\right) x^{2} \in I$. Also $\left(c^{2^{n}-1}+c^{2^{n}-2}\right) c^{2^{n}-2} x^{2^{n}-2}+\cdots+\left(c^{2^{n}-1}+c^{2}\right) c^{2} x^{2}=$ $\left(\left(c^{2^{n}-1}+c^{2^{n}-2}\right) x^{2^{n}-2}+\cdots+\left(c^{2^{n}-1}+c^{2}\right) x^{2}\right) \circ(c x) \in I$ and $\left(c^{2^{n}-1}+c^{2^{n}-2}\right) c^{2^{n}-2} x^{2^{n}-2}+$ $\cdots+\left(c^{2^{n}-1}+c^{2}\right) c^{2^{n}-2} x^{2}=\left(c^{2^{n}-2} x\right) \circ\left(\left(c^{2^{n}-1}+c^{2^{n}-2}\right) x^{2^{n}-2}+\cdots+\left(c^{2^{n}-1}+c^{2}\right) x^{2}\right) \in I$. Hence $\left(c^{2^{n}-1}+c^{2^{n}-3}\right)\left(e^{2^{n}-2}+c^{2^{n}-3}\right) x^{2^{n}-3}+\cdots+\left(c^{2^{n}-1}+c^{2}\right)\left(c^{2^{n}-2}+c^{2}\right) x^{2} \in I$. If we continue this procedure, we finally arrive at $\left(c^{2^{n}-1}+c^{2}\right)\left(c^{2^{n}-2}+\right.$ $\left.c^{2}\right) \cdots\left(c^{3}+c^{2}\right) x^{2} \in I$ where the coefficient of $x^{2} \neq 0$. So $x^{2} \in I$ and we get $I=\left(\left(x^{2}\right)\right)$ again by Lemma 14 .

Our last case is $R=Z_{2}$. This case is rather complicated and so the way is longer. Brenner has shown in [1] that there are only two maximal ideals in $\boldsymbol{Z}_{2}[x]$. One of them is $T:=$ the subgroup generated by $\left\{1, x+x^{2}, x^{3}, x+x^{4}, x+x^{5}, x^{6}, x+x^{7}, x+x^{8}, x^{9}, \cdots\right\}$. The other one is $V$, the subgroup generated by $\left\{1, x+x^{2}, x+x^{3}\right.$, $\left.x+x^{4}, \cdots\right\}$. We define $T_{0}, V_{0}$ as follows: $T_{0}:=T \cap\left(\boldsymbol{Z}_{2}\right)_{0}[x]$ and $V_{0}:=$ $V \cap\left(\boldsymbol{Z}_{2}\right)_{0}[x] . \quad T_{0}$ and $V_{0}$ are easily shown to be ideals in $\left(\boldsymbol{Z}_{2}\right)_{0}[x]$. They are even strictly maximal ideals as will be demonstrated in the following. Together with $\left(\left(x^{2}\right)\right)$, there are just three strictly maximal ideals in $\left(\boldsymbol{Z}_{2}\right)_{0}[x]$.

LeMma 18. Let $I$ be a strictly maximal ideal in $\left(\boldsymbol{Z}_{2}\right)_{0}[x]$ with $x^{2} \in I$, then $I=\left(\left(x^{2}\right)\right)$.

Proof. Since $x^{2} \in I, x^{2 k}=x^{2} \circ x^{k} \in I$ for all $k \in N$. Hence $\left(x^{4}+x\right)^{3}+$ $x^{3} \in I$, whence $x^{9} \in I$. But $x^{9}=x^{3} \circ x^{3}$ so $x^{3} \in I$ since $\left(Z_{2}\right)_{0}[x] / I$ has no divisors of zero. Therefore $x^{6 k}+x^{4 k+3}+x^{2 k+6}+x^{9}=\left(x^{2 k}+x^{3}\right)^{3} \in I$, from which we get that $x^{4 k+3} \in I$ for all $k \in N$. Also, $\left(x^{2 k}+x\right)^{3}+x^{3} \in I$ gives us $x^{4 k+1} \in I$ for all $k \in N$. All $x^{4}$ and $x^{4 k+2}=x^{2} \circ x^{2 k+1}$ are also in $I$, so, putting altogether, $x^{n} \in I$ for $n \geqq 2$, which means $I=\left(\left(x^{2}\right)\right)$.

LEMMA 19. Let $I$ be a strictly maximal ideal in $\left(\boldsymbol{Z}_{2}\right)_{0}[x]$ with $x^{2} \notin I, x^{3} \in I$. Then $I=T_{0}$

Proof. By Lemma 16 and the information in the proof of Lemma 17, we know $\left(\boldsymbol{Z}_{2}\right)_{0}[x] / I$ is a finite Dickson near-field of characteristic 2 , so it has order $2^{t}$ (by 8.13 of [3]). Since $x^{2}+I \neq 0+I$, the order $k$ of $x^{2}+I$ divides $2^{t}-1$. So we have $x^{2 k}+I=\left(x^{2}+I\right) \circ\left(x^{2}+I\right) \circ \cdots \circ$ $\left(x^{2}+I\right)=x+I$ and $k / 2^{t}-1$. Hence $k$ is odd, whence $3 / 2^{k}+1$. Let $2^{k}+1=: 3 j$. For all $s \in N, s \geqq 3$, we get $x^{3} \circ\left(x^{s}+x^{s-1}\right) \in I$ whence $x^{3 s-1}+x^{3 s-2} \in I$ and $x^{3} \circ\left(x^{s}+x^{s-2}\right) \in I$ whence $x^{3 s-2}+x^{3 s-4} \in I$. Hence $x^{3 s-1} \equiv x^{3 s-2} \equiv x^{3 s-3} \equiv x^{3 s-5} \equiv \cdots \equiv x^{5} \equiv x^{4}(\bmod I)$. In particular, $x \equiv$ $x^{2^{k}}=x^{3 j-1} \equiv x^{4}$ and we get $x^{n}+x \in I$ for all $n \in N, 3 \nmid n, n \geqq 4$. Also, from $\left(x^{2}+I\right) \circ\left(x^{2}+I\right)=x^{4}+I=x+I$ we get $x^{2}+I=x+I$ by 8.10.a of [3]. Hence all the additive generators of $T_{0}$ are in $I$, whence $T_{0} \subseteq I$. But $T_{0}$ is a subgroup of $\left(Z_{2}\right)_{0}[x]$ of order 2 , hence $T_{0}=I$. 
LEMMA 20. Let $I$ be a strictly maximal ideal of $\left(\boldsymbol{Z}_{2}\right)_{0}[x]$ with $x^{2} \notin I, x^{3} \notin I, x^{2}+x^{3} \in I$. Then $I=V_{0}$.

Proof. Since $x^{2}+x^{3} \in I$, also $\left(x^{2}+x^{3}\right) \circ\left(x^{s}+x\right) \in I$, whence $x^{2 s+1}+$ $x^{s+2} \in I$ and $\left(x^{2}+x^{3}\right) \circ\left(x^{s}+x^{2}\right) \in I$, implying that $x^{2 s+2}+x^{8+4} \in I$. From the first result we get $x^{5} \equiv x^{4}, x^{7} \equiv x^{5}, x^{9} \equiv x^{6}(\bmod I)$ and from the second we derive $x^{8} \equiv x^{7}, x^{10} \equiv x^{8}, x^{12} \equiv x^{9}, \cdots(\bmod I)$, so (since also $\left.\left(x^{2}+x^{3}\right) \circ x^{2}=x^{4}+x^{6} \in I\right)$ we get $x^{4} \equiv x^{5} \equiv x^{6} \equiv \cdots(\bmod I)$. Since $x^{2} \notin I$, there is some $k \in N$ with $x^{2^{k}}+x \in I$ (same reason as in the proof of Lemma 19). Hence $x \equiv x^{2^{k}} \equiv x^{4}(\bmod I)$. Also $\left(x^{2^{k}}+x\right) \circ x^{2} \in I$, whence $x^{2} \equiv x^{2^{k+1}} \equiv x^{4}(\bmod I)$. Since $x^{2}+x^{3} \in I$, we get $x^{2} \equiv x^{3}(\bmod$ $I)$, and therefore $x \equiv x^{2} \equiv x^{3} \equiv x^{4} \equiv \cdots \equiv x^{n} \equiv \cdots(\bmod I)$. Thus for all $n \in N x^{n}+x \in I$, hence $V_{0} \subseteq I$. But $V_{0}$ is a subgroup of index 2 in $\left(Z_{2}\right)_{0}[x]$, so $V_{0}=I$.

Lemma 21. Let $I$ be a strictly maximal ideal of $\left(\boldsymbol{Z}_{2}\right)_{0}[x]$. Then $I$ is either $=\left(\left(x^{2}\right)\right)$ or $=T_{0}$ or $=V_{0}$.

Proof. Suppose $I \neq\left(\left(x^{2}\right)\right), I \neq T_{0}, I \neq V_{0}$. Applying Lemmas 18, 19 and 20 we have: $x^{2} \notin I, x^{3} \notin I, x^{2}+x^{3} \notin I$. As in the proof of Lemma 17 , let $C(K)$ be the center of $K:=\left(\boldsymbol{Z}_{2}\right)_{0}[x] / I$. Obviously $x+I \in C(K), x^{2}+I \in C(K)$, hence $x+I+x^{2}+I=x+x^{2}+I \in C(K)$. So $\left(x^{2}+x+I\right) \circ\left(x^{3}+I\right)=\left(x^{3}+I\right) \circ\left(x^{2}+x+I\right)$, hence $x^{6}+x^{3}+I=$ $x^{6}+x^{5}+x^{4}+x^{3} \in I$ and $x^{5}+x^{4} \in I$. Also, $\left(x^{5}+x^{4}\right) \circ\left(x^{2}+x\right)=x^{10}+$ $x^{9}+x^{6}+x^{5}+x^{8}+x^{4} \in I$. Since $\left(x^{5}+x^{4}\right) \circ x^{2}=x^{10}+x^{8} \in I$ and $x^{5}+x^{4} \in I$, we have $x^{9}+x^{6} \in I$. But $I=x^{9}+x^{6}+I=\left(x^{3}+x^{2}+I\right) \circ\left(x^{3}+I\right)$, implying that either $x^{3}+x^{2} \in I$ or $x^{3} \in I$, both being contradictions.

Lemma 22. Let $I$ be a strictly maximal ideal of $\left(\boldsymbol{Z}_{2}\right)_{0}[x]$. Then $\left(\boldsymbol{Z}_{2}\right)_{0}[x] / I \cong \boldsymbol{Z}_{2}$.

Proof. Applying Lemma 21, we know $I$ is either $=\left(\left(x^{2}\right)\right)$ or $=T_{0}$ or $=V_{0}$. But $\left[\left(\boldsymbol{Z}_{2}\right)_{0}[x]:\left(\left(x^{2}\right)\right)\right]=\left[\left(\boldsymbol{Z}_{2}\right)_{0}[x]: T_{0}\right]=\left[\left(\boldsymbol{Z}_{2}\right)_{0}[x]: V_{0}\right]=2$. So we have in all of these three cases: $\left(\boldsymbol{Z}_{2}\right)_{0}[x] / I \cong \boldsymbol{Z}_{2}$.

This completes the proof of Theorem 1 .

As a byproduct, we have a complete knowledge of all strictly maximal ideals in polynomial near-rings:

CoRollary. Let $I$ be a strictly maximal ideal of $R_{0}[x]$. Then there exists a maximal ideal $M$ of $R$ with $I=\left(\left(x^{2}\right)\right)+M x$, unless $R=Z_{2}$. In this case, I might as well be $=T_{0}$ or $=V_{0}$. 
In particular, for a field $R \neq \boldsymbol{Z}_{2}$, there is just one strictly maximal ideal, namely $\left(\left(x^{2}\right)\right)$.

G. Pilz suggested to investigate near-fields which are contained in $R[x]$. Since all near-fields with the exception of a trivial one ([3], 8.1-we exclude this one from our considerations) are zero-symmetric, we only need to search them in $R_{0}[x]$.

Lemma 23. Let $R$ be an integral domain and $F$ a near-field in $R_{0}[x]$. Then there is a subfield $K$ of $R$ such that $F=\{a x / a \in K\}$.

Proof. Straightforward.

Lemma 24. Let $F$ be a near-field in $R_{0}[x], 0 \neq f=a_{n} x^{n}+\cdots+$ $a_{1} x \in F$. Then $a_{2}, a_{3}, \cdots, a_{n} \in \mathfrak{P}(R)$ (prim-radical of $R$ ) and $a_{1}$ is a unit in $R$.

Proof. We use the following epimorphisms: $h: R \rightarrow R / M$ where $M$ is a prime ideal of $R, h^{\prime}: R_{0}[x] \rightarrow(R / M)_{0}[x]:$

$$
a_{n} x^{n}+\cdots+a_{1} x \longmapsto h\left(a_{n}\right) x^{n}+\cdots+h\left(a_{1}\right) x .
$$

In $(R / M)_{0}[x]$ we can apply Lemmas 2, 3 and get: $h\left(a_{2}\right)=h\left(a_{3}\right)=\cdots=$ $h\left(a_{n}\right)=0$. So we have $a_{2}, \cdots, a_{n} \in \mathfrak{P}(R)$.

Since $f \neq 0, a_{1}$ cannot be $=0$, otherwise $f$ has no inverse in $F$.

Suppose $a_{1}$ were not a unit, so $a_{1}$ is in a maximal ideal $M_{1}$ of $R$. Let $h: R \rightarrow R / M_{1}$ and $h^{\prime}: R_{0}[x] \rightarrow\left(R / M_{1}\right)_{0}[x]$ be as above and we get $h^{\prime}\left(a_{n} x^{n}+\cdots+a_{1} x\right)=h\left(a_{1}\right) x=0$, a contradiction to the fact that $h^{\prime}(F)=\{a x / a \in K\}$ for some subfield $K$ of $h(R)$.

TheOREM 2. Let $F$ be a near-field contained in $R_{0}[x], F_{1}:=$ $\left\{a_{1} /\right.$ some $\left.a_{n} x^{n}+\cdots+a_{1} x \in F\right\}$. Then $F \cong F_{1} x$.

Proof. Define $h: F \rightarrow F_{1} x$.

$$
a_{n} x^{n}+\cdots+a_{1} x \longmapsto a_{1} x
$$

$h$ is surjective. We show it is injective, too. Let $f_{1}, f_{2} \in F$ with $f_{1}=a_{n} x^{n}+\cdots+a_{1} x$ and $f_{2}=b_{m} x^{m}+\cdots+a_{1} x$. Then $f_{1}-f_{2}=\cdots+$ $\left(a_{2}-b_{2}\right) x^{2}+0 x \in F$. But then $f_{1}-f_{2}=0$ by Lemma 24. Hence $f_{1}=f_{2}$ and $h$ is $1-1$.

It is easy to show that $h$ is a near-ring homomorphism, so $h$ is a near-ring isomorphism.

ExAmples. Take $R:=Z_{2}[t] /\left(t^{4}+t^{2}+1\right)$. Then $K_{1}:=\{0, x\}, K_{2}:=$ $\left\{0, x, t^{2} x,\left(t^{2}+1\right) x\right\}$ and $K_{3}:=\left\{0, x,\left(t^{2}+t+1\right) x^{2}+t^{2} x,\left(t^{2}+t+1\right) x^{2}+\right.$ 
$\left.\left(t^{2}+1\right) x\right\}$ are examples of subnear-fields of $R_{0}[x]$. Note that $K_{3}$ contains non-liear polynomials.

Application. Let $P$ be a planar near-ring with identity which is either contained in some $R_{0}[x]$ or a factor of $R_{0}[x]$. Then $P$ is a field and isomorphic to a subfield or a factorfield of $R$. This holds because a planar near-ring with identity is accurately a near-field, as can be easily seen.

\section{REFERENCES}

1. J. L. Brenner, Maximal ideals in the near-ring of polynomials mod 2, Pacific J. Math., 52 (1974), 595-600.

2. J.R. Clay and D. K. Doi, Maximal ideals in the near-ring of polynomials over a field. In Colloqu. Math. Soc. Janus Bolyai 6, Rings, Modules and Radicals (ed. by A. Kertesz), pp. 117-133. Amsterdam, North Holland, 1973.

3. G. Pilz, Near-rings, Amsterdam, North Holland, 1977.

4. Y. S. So, Polynom Fast-ring, Dissertation, Univ. Linz, 1978.

5. E. G. Straus, Remarks on the paper "Ideals in near-rings of polynomials over a field”, Pacific J. Math., 52 (1974), 601-603.

6. H. Wähling, Bericht über Fastkörper, Jahresbericht Dt. Math. Ver., 76 (1975), 41-103.

Received December 15, 1979. I would like to thank Günter Pilz for his unselfish help and encouragement and to "Österreichischen Forschungsfonds" for the financial assistance (Project number 3479).

INSTITUTE FÜR MATHEMATIK

JOHANNES-KEPLER UNIVERSITÄT

A-4045 Linz-Aunof, Austria. 



\section{PACIFIC JOURNAL OF MATHEMATICS}

\section{EDITORS}

DONALD BABBITT (Managing Editor)

University of California

Los Angeles, California 90024

Hugo RossI

University of Utah

Salt Lake City, UT 84112

C. C. MOORE and ANDREW OGG

University of California

Berkeley, CA 94720

\section{J. DugundJI}

Department of Mathematics University of Southern California Los Angeles, California 90007

R. FinN and J. Milgram Stanford University Stanford, California 94305

\section{ASSOCIATE EDITORS}
R. ARENS
E. F. BECKENBACH
B. H. Neumann
F. WOLF
K. YoshidA

\section{SUPPORTING INSTITUTIONS}

UNIVERSITY OF ARIZONA

UNIVERSITY OF BRITISH COLUMBIA

CALIFORNIA INSTITUTE OF TECHNOLOGY

UNIVERSITY OF CALIFORNIA

MONTANA STATE UNIVERSITY

UNIVERSITY OF NEVADA, RENO

NEW MEXICO STATE UNIVERSITY

OREGON STATE UNIVERSITY
UNIVERSITY OF OREGON

UNIVERSITY OF SOUTHERN CALIFORNIA

STANFORD UNIVERSITY

UNIVERSITY OF HAWAII

UNIVERSITY OF TOKYO

UNIVERSITY OF UTAH

WASHINGTON STATE UNIVERSITY

UNIVERSITY OF WASHINGTON 


\section{Pacific Journal of Mathematics}

\section{Vol. 96, No. $1 \quad$ November, 1981}

Hédi Amara, Groupe des classes et unité fondamentale des extensions quadratiques relatives à un corps quadratique imaginaire principal $\ldots \ldots \ldots 1$

Douglas S. Bridges, On the isolation of zeroes of an analytic function $\ldots \ldots 13$ Andrew J. Casson and John L. Harer, Some homology lens spaces which

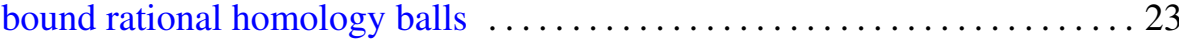

Z. A. Chanturia, On the absolute convergence of Fourier series of the classes $H^{\omega} \cap V[v]$

J.-F. Colombeau and Mário Carvalho Matos, On some spaces of entire functions defined on infinite-dimensional spaces $\ldots \ldots \ldots \ldots \ldots \ldots \ldots 63$

Edwin Duda, Pointwise periodic homeomorphisms on chainable continua . . .77

Richard F. Gustafson, A simple genus one knot with incompressible spanning surfaces of arbitrarily high genus $\ldots \ldots \ldots \ldots \ldots \ldots \ldots \ldots 1$

Fumio Hiai, Masanori Ohya and Makoto Tsukada, Sufficiency, KMS condition and relative entropy in von Neumann algebras

Ted Hurley, Intersections of terms of polycentral series of free groups and free Lie algebras. II .................................. 111

Robert Edward Jamison, II, Partition numbers for trees and ordered sets . . 115 R. D. Ketkar and N. Vanaja, A note on FR-perfect modules ............. 141 Michihiko Kikkawa, On Killing-Ricci forms of Lie triple algebras ....... 153

Jorge Lewowicz, Invariant manifolds for regular points 163

Richard W. Marsh, William H. Mills, Robert L. Ward, Howard Rumsey and Lloyd Richard Welch, Round trinomials .....

Claude Schochet, Topological methods for $C^{*}$-algebras. I. Spectral sequences

Yong Sian So, Polynomial near-fields?

Douglas Wayne Townsend, Imaginary values of meromorphic functions in the disk

Kiyoshi Watanabe, Coverings of a projective algebraic manifold .. 243

Martin Michael Zuckerman, Choosing $l$-element subsets of $n$-element sets 